\title{
Diagnostic importance of 9p21 homozygous deletion in malignant mesotheliomas
}

\author{
Simion Chiosea ${ }^{1}$, Alyssa Krasinskas ${ }^{1}$, Philip T Cagle², Kisha A Mitchell ${ }^{1}$, Dani S Zander \\ and Sanja Dacic ${ }^{1}$ \\ ${ }^{1}$ Division of Anatomic Pathology, Department of Pathology, University of Pittsburgh Medical Center, \\ Presbyterian University Hospital, Pittsburgh, PA, USA; ${ }^{2}$ The Department of Pathology, The Methodist \\ Hospital, Houston, TX, USA and ${ }^{3}$ Department of Pathology, Penn State Milton S. Hershey Medical Center, \\ Hershey, PA, USA
}

\begin{abstract}
Definitive diagnosis of malignant mesothelioma in small specimens can be extremely difficult based on morphology alone. Homozygous deletion of 9p21, the locus harboring the p16 gene, has been reported as the most common genetic alteration in malignant mesotheliomas. Recent studies demonstrated that this alteration may be useful for differentiating benign from malignant mesothelial proliferations in cytology specimens. The aim of this study was to evaluate the diagnostic utility of homozygous deletion of 9p21 assessed by fluorescence in situ hybridization (FISH) in mesothelial proliferations involving serosal surfaces in paraffinembedded tissue. $p 16$ protein immunoexpression was also explored as a potential diagnostic aid. FISH analysis demonstrated homozygous deletion of the 9p21 locus in 35 of 52 cases $(67 \%)$ of pleural mesothelioma and in 5 of 20 cases of peritoneal mesothelioma $(25 \%)(P<0.005)$. None of 40 cases of reactive pleural mesothelial proliferations showed $p 16$ deletion $(P<0.005)$. Loss of immunoexpression of $p 16$ was observed in $71 \%$ of the peritoneal mesotheliomas, $40 \%$ of the pleural malignant mesotheliomas and $15 \%$ of the reactive mesothelial cells. Homozygous deletion did not correlate with p16 protein expression in any of the studied groups. Our study suggests that 9p21 homozygous deletion assessed by FISH on paraffin-embedded tissue may be helpful for differentiating between malignant mesotheliomas and reactive mesothelial proliferations. A discrepancy between p16 protein expression and homozygous deletion suggests that other molecular mechanisms may play a role in p16 protein expression in mesothelial proliferations.

Modern Pathology (2008) 21, 742-747; doi:10.1038/modpathol.2008.45; published online 7 March 2008
\end{abstract}

Keywords: mesothelioma; 9p21; p16; immunohistochemistry; FISH

A number of international panels of experts have determined criteria for the diagnosis of diffuse malignant mesothelioma, including the United States Canadian Mesothelioma Reference Panel, the World Health Organization Consensus Conference; the International Mesothelioma Panel (Programme National De Surveillance Des Mesotheliomes, The Institut de la Veille Sanitaire, Ministry of Health, France) and the International Mesothelioma Interest Group (IMIG). ${ }^{1-5}$ These expert panels assert that most diffuse malignant mesothelioma can be recognized or strongly suspected on routine hematoxylin and eosin (H\&E) stain of serosal membrane biopsies. ${ }^{1-5}$ Diagnosis of diffuse malignant mesothelioma is

Correspondence: Dr S Dacic, MD, PhD, Department of Pathology-PUH A610, University of Pittsburgh Medical Center, Presbyterian University Hospital, 200 Lothrop Street, Pittsburgh, PA 15213, USA.

E-mail: dacics@upmc.edu

Received 26 March 2007; revised 27 October 2007; accepted 31 October 2007; published online 7 March 2008 based primarily on a correlation of histopathology with clinical and imaging information and gross impressions of the surgeon. Diffuse malignant mesothelioma exhibit a wide range of histopathologic patterns that may potentially mimic other types of cancers and vice versa. ${ }^{6-8}$ Since clinical, radiologic and gross features of other types of cancer may also resemble diffuse malignant mesothelioma, immunohistochemistry is often used to provide additional support for the diagnosis of diffuse malignant mesothelioma $v s$ a variety of other types of cancer that might be primary or metastatic to the serosal membranes. Generally, two antibodies that are positive markers for diffuse malignant mesothelioma and two antibodies that are negative markers for diffuse malignant mesothelioma are recommended for immunohistochemical confirmation of the diagnosis. Additional antibodies are used according to the differential diagnosis under consideration or because initial immunostaining results are equivocal. ${ }^{9-11}$ Expert panels generally refrain from advocating a specific set of antibodies to allow 
for individual flexibility, but most institutions have a routine group of antibodies that they produce for the differential diagnosis of diffuse malignant mesothelioma.

On the other hand, distinction between benign reactive mesothelial proliferations and diffuse malignant mesothelioma on serosal membrane biopsies is an occasional, but well-known diagnostic dilemma for surgical pathologists that has serious implications for the patient: a diagnosis of diffuse malignant mesothelioma means certain death and sometimes attempts at radiation and/or chemotherapy, radical surgery and/or novel therapies, all with significant morbidity. ${ }^{12,13}$ There are a large number of common and relatively common conditions that can produce reactive mesothelial hyperplasia and are often self-limited or readily treatable, including infections, drug reactions, pulmonary infarcts, pneumothorax, collagen vascular diseases, surgery and trauma. Reactive mesothelial proliferations may have histologic features that resemble malignancy, including increased cellularity, cytologic atypia and mitoses, architectural atypia such as papillary excrescences and lumens and pseudoinvasion (entrapment). A variety of histologic features favor malignancy, including tumor nodules, bland necrosis, full thickness cellularity and, especially, frank invasion of underlying lung parenchyma or fat. In most cases, the correlation of clinical, radiologic and gross findings is helpful in diagnosis, but rarely reactive processes may grossly resemble malignancy. More frequently of concern to the surgical pathologist is the surgical specimen that is suspected of harboring an early diffuse malignant mesothelioma with minimal gross findings. As noted in the literature, very early diffuse malignant mesothelioma with minimal invasion may occasionally be sampled as an incidental or fortuitous finding in surgical specimens in which diffuse malignant mesothelioma is not clinically suspected. ${ }^{14}$ Other lesions that are presumed to exist, but currently lack reliable criteria for independent diagnosis, are premalignant and preinvasive mesothelial neoplasms, including mesothelioma-in-situ, which could presumably be sampled without gross features of malignancy., ${ }^{3,45-17}$ These concepts and their diagnostic criteria are currently under investigation by the International Mesothelioma Panel, which has recommended the term 'atypical mesothelial proliferation' for equivocal cases of benign mesothelial hyperplasia vs diffuse malignant mesothelioma. ${ }^{4}$

For over 15 years, multiple investigators have attempted to identify a reliable biomarker for distinguishing diffuse malignant mesothelioma from benign reactive mesothelial proliferations with immunostains for $p 53$ product, epithelial membrane antigen and desmin as primary candidates, but no satisfactorily reproducible biomarker has yet been confirmed. ${ }^{18-23}$ Recently, the International Mesothelioma Panel has undertaken investigation of potential biomarkers for this purpose. A recent report suggests that glucose transporter (GLUT)-1, member of the mammalian facilitative GLUT family of passive carriers, may be a potential marker for malignancy. ${ }^{24}$

One of the most common genetic alterations in mesothelioma is the homozygous deletion of the 9p21 locus within a cluster of genes that includes CDKN2A, CDKN2B and MTAP. ${ }^{25-28}$ Several cytogenetic and molecular studies have reported p16/ CDKN2A deletions in up to $72 \%$ of primary mesotheliomas. ${ }^{29,30}$ Thus, detection of homozygous $p 16 /$ CDKN2A deletion by fluorescence in situ hybridization (FISH) seems to be feasible and helpful in confirming a diagnosis of mesothelioma in cytologic and surgical specimens. This observation opened a new avenue for surgical pathologists in resolving the diagnostic dilemmas in mesothelial pathology.

The aim of our study was to determine the potential diagnostic utility of FISH detection of the homozygous deletion of the 9p21 locus for evaluating the nature of mesothelial proliferations in surgical specimens. We also compared the frequency of this deletion in mesothelioma from two different anatomic sites, the pleura and peritoneum. We also explored the status of $p 16$ by immunohistochemistry and compared the two methods.

\section{Materials and methods}

Fifty-two pleural malignant mesotheliomas (28 cases on tissue microarray, 24 whole sections), 21 peritoneal malignant mesotheliomas and 40 cases of benign acute and chronic pleuritis were obtained from the paraffin block archives of the University of Pittsburgh Medical Center, Penn State Milton S Hershey Medical Center, The Methodist Hospital, Houston, TX, USA, and the consultation files of one of us (PC). The study was approved by the University of Pittsburgh institutional review board (IRB no. 0612074) and the Patient Advocacy Council Institutional Review Board (PAC no. 7-0013-04 and PAC no. 7-0014-04). The diagnosis of diffuse malignant mesothelioma was based on the combination of clinical findings, imaging and gross observations at surgery and routine H\&E histology. The diagnosis was confirmed by an immunohistochemistry panel that is used routinely at UPMC (calretinin, WT-1, D2-40, cytokeratin 5/6, Ber-EP4, B72.3, TTF-1, p63, CEA). Representative tissue blocks were selected for $p 16$ immunostaining and FISH analysis.

High-density tissue microarrays were constructed from archival formalin-fixed, paraffin-embedded samples of 42 mesotheliomas obtained at The Methodist Hospital, Houston, TX, USA. For each sample, areas rich in tumor cells were identified by light microscopic examination of $\mathrm{H} \& \mathrm{E}$ sections and selected for use in the tissue microarrays. Three cores measuring $0.1 \mathrm{~cm}$ in diameter were taken from the donor paraffin tissue blocks of each case and were arranged in a recipient paraffin tissue array block by using a Manual Tissue Arrayer (MTA-1, Beecher Instruments Inc., Sun Prairie, WI, USA). 


\section{Immunohistochemistry}

The immunohistochemical study was performed using anti-p16 mouse monoclonal antibody at a dilution of 1:200 (BD PharMingen, San Diego, CA, USA) according to the standard avidin-biotinperoxidase complex method. The $p 16$ immunostain for each case was reviewed independently by two investigators (AK and SD) who had no knowledge of the FISH findings and was interpreted as described previously. ${ }^{31}$ Nuclear staining with or without cytoplasmic staining in $>10 \%$ of targeted cells was interpreted as positive. Cases that demonstrated no nuclear staining were considered negative. Sections of cervical biopsy previously identified as positive for p16 protein served as a positive control. Negative controls included omitting the primary antibody and its substitution with normal serum.

\section{Fluorescence In Situ Hybridization}

Dual-color FISH analysis was performed using a Spectrum Green-labeled chromosome 9 centromeric probe and a Spectrum-Orange labeled, locus-specific CDKN2A (p16) probe (Vysis, Downers Grove, IL, USA). ${ }^{32}$ In brief, paraffin sections were de-paraffinized, dehydrated in ethanol and air-dried. Sections were digested with protease $\mathrm{K}(0.5 \mathrm{mg} / \mathrm{ml})$ at $37^{\circ} \mathrm{C}$ for $28 \mathrm{~min}$. The slides were denatured at $75^{\circ} \mathrm{C}$ for $5 \mathrm{~min}$ in $70 \%$ Formamide (Chemicon, Billerica, MA, USA) and dehydrated in ethanol. The probes were denatured for $5 \mathrm{~min}$ at $75^{\circ} \mathrm{C}$ prior to hybridization. Slides were hybridized overnight at $37^{\circ} \mathrm{C}$ and washed in 2XSSC/0.3\% Igepal (Sigma, St Louis, MO, USA) at $72{ }^{\circ} \mathrm{C}$ for $2 \mathrm{~min}$. Nuclei were counterstained with DAPI/antifade (Vysis Inc., Downers Grove, IL, USA). Each FISH assay included normal lung tissue sections as a negative control, and sections of non-small cell lung carcinoma previously identified as carrying $p 16$ deletion as a positive control. Analyses were performed using a fluorescence microscope (Nikon Eclipse E600) and Cytovysion Workstation (Applied Imaging, Santa Clara, CA, USA) equipped with filter sets with single and dual band excitors for Spectrum Green, Spectrum Orange and DAPI (UV $360 \mathrm{~nm}$ ). The histological areas previously selected on the H\&E-stained sections were identified on the FISH-treated slides. Only individual and well-delineated cells were scored. Overlapping cells were excluded from the analysis. At least 60 cells were scored for each case and control.

Each tumor was assessed by the average and the maximum numbers of copies of $p 16$ gene per cell and the average ratio of $p 16$ gene to chromosome 9 copy numbers (CEP9). Homozygous deletion was defined if both 9p21 signals were lost in at least $20 \%$ of nuclei and showed at least one signal for the CEP-9 probe. ${ }^{26}$

\section{Statistical Analysis}

Fisher's exact probability test was used to compare the rates of positives between the groups. All
$P$-values were adjusted for multiple comparisons using a Bonnferroni correction at $P<0.05$.

\section{Results}

The results of $p 16$ expression assessed by immunohistochemistry and p16 deletion assessed by FISH are shown in Table 1. FISH analysis demonstrated homozygous deletion of 9p21 locus in 35 of 52 cases $(67 \%)$ of pleural mesothelioma. There was no difference between different histologic subtypes of mesothelioma. Homozygous deletion of 9p21 was less frequent in peritoneal mesothelioma and found in only 5 of 20 cases (25\%). The difference between the two mesothelioma groups was significant $(P<0.005)$ (Figures 1 and 2a). Cases positive for deletion showed homozygous deletion of 9p21 in 90-100\% of analyzed cells. Hemizygous loss was not identified in any of analyzed cases of malignant mesotheliomas. No 9p21 deletion was detected in any of 40 reactive mesothelial proliferations associated with acute or chronic pleuritis $(P<0.005)$.

Also noted on FISH analysis, chromosome 9 copy number was abnormal in 18 cases (16\%), including $10(19 \%)$ pleural mesotheliomas and $8(40 \%)$ peritoneal mesotheliomas. Polyploidy was more frequent (12 cases) than monosomy (6 cases).

Table 1 Summary of p16 immunohistochemical and FISH analysis

\begin{tabular}{|c|c|c|}
\hline Study group & $\begin{array}{c}\text { Immunohistochemistry } \\
\text { p16 positive (\%) }\end{array}$ & $\begin{array}{c}\text { FISH p16 deletion } \\
(\%)\end{array}$ \\
\hline Pleural mesothelioma & $32 / 52(60 \%)$ & $35 / 52(67 \%)$ \\
\hline $\begin{array}{l}\text { Peritoneal } \\
\text { mesothelioma }\end{array}$ & $6 / 21(29 \%)$ & $5 / 20(25 \%)$ \\
\hline Pleuritis & $34 / 40(87 \%)$ & $0 / 40(0 \%)$ \\
\hline
\end{tabular}

Abbreviations: FISH, fluorescence in situ hybridization; IHC, immunohistochemistry.

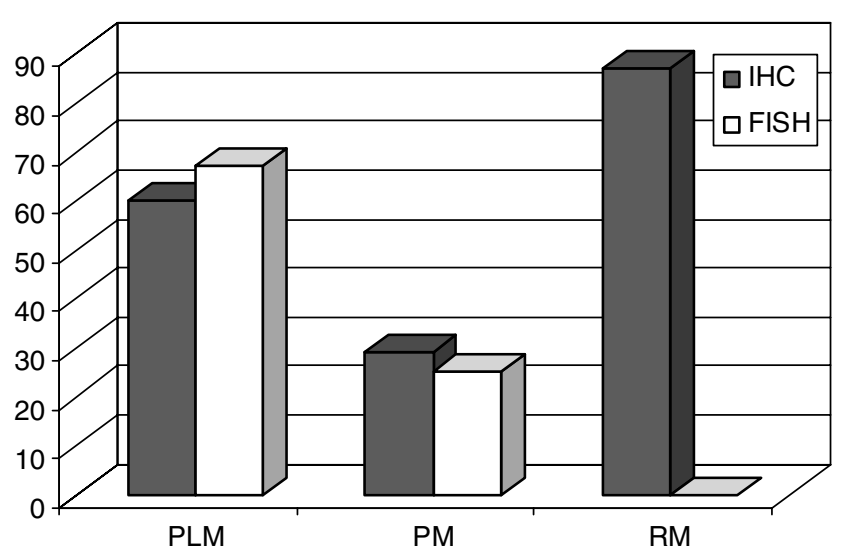

Figure 1 Frequency of homozygous deletion of 9p21 and loss of p16 expression in benign mesothelial proliferations and malignant mesothelioma. (IHC, immunohistochemistry; FISH, fluorescence in situ hybridization; PLM, pleural malignant mesothelioma; PM, peritoneal mesothelioma; RM, reactive mesothelial cells). 

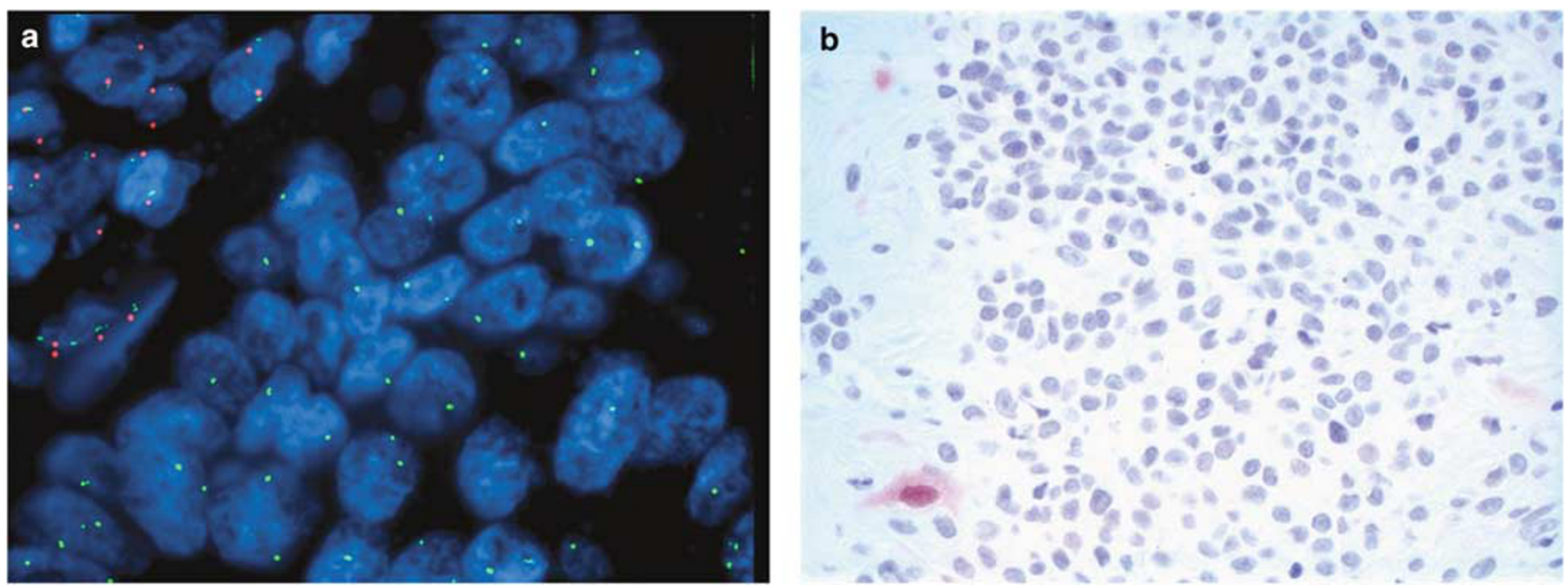

Figure 2 (a) Example of malignant mesothelioma with homozygous deletion of 9p21 by FISH with a CDKN2A ( $p 16$ ) (orange) and chromosome 9 (green)-specific probe (magnification $\times 630$ ) and $(\mathbf{b})$ loss of p16 protein expression by immunohistochemistry. Strong nuclear expression of p16 in reactive mesothelial cell, but not in malignant mesothelioma, is shown (original magnification $\times 40$ ).

Different tissue preparations, whole sections vs tissue microarrays, did not have impact on the interpretation of FISH results. It seems that FISH tissue microarray is a good tool for analysis of a large number of mesotheliomas. This could be explained, in part, by the fact that FISH analysis on a whole section includes only small targets that contain similar number of cells present in the individual tissue microarray cores.

Immunohistochemical $p 16$ positivity was observed in all study groups. Both benign and malignant pleural mesothelial lesions showed p16 expression ( 87 vs $60 \%$ ), and the difference was not statistically significant. Peritoneal mesotheliomas were positive in $29 \%$ of cases. The difference between the peritoneal mesotheliomas and reactive mesothelial proliferations was significant $(P=$ 0.001) (Figures 1 and 2b). Similar to the FISH results, the expression of $p 16$ was independent of the histologic subtype of malignant mesothelioma. Discrepancies in number of cases analyzed by immunohistochemistry and FISH assay reflect unsuccessful hybridization.

Table 2 summarizes the results of 9p21 homozygous deletion determined by FISH in comparison with $p 16$ expression determined by immunohistochemistry. Statistical analysis demonstrates that the two methods, overall, show little correlation. Briefly, pleural malignant mesotheliomas showed a trend towards a good correlation between homozygous deletion of $9 \mathrm{p} 21$ and loss of $p 16$ protein expression. Cases with the 9p21 deletion tended to lose $p 16$ expression, while cases without a 9p21 deletion tended to maintain intact $p 16$ expression; however, this was not statistically significant $(P=0.06)$. A similar trend was noted in cases of peritoneal mesotheliomas $(P=0.26)$ but not in reactive benign pleural mesothelial proliferations $(P=1.0)$.
Table 2 Comparison of p16 expression determined by immunohistochemistry and 9p21 homozygous deletion determined by FISH in benign and malignant mesothelial proliferations

\begin{tabular}{lrrrrr}
\hline & \multicolumn{4}{c}{ IHC/FISH } & \\
\cline { 2 - 4 } Study group & $-/-$ & $-/+$ & $+/-$ & $+/+$ & P-value \\
\hline Pleural mesothelioma $(N=52)$ & 2 & 18 & 15 & 17 & 0.06 \\
Peritoneal mesothelioma $(N=20)$ & 9 & 5 & 6 & 0 & 0.26 \\
Pleuritis $(N=40)$ & 6 & 0 & 34 & 0 & 1.0
\end{tabular}

Abbreviations: FISH, fluorescence in situ hybridization; IHC, immunohistochemistry.

\section{Discussion}

Prior cytogenetic and molecular studies demonstrated homozygous deletion of the 9p21 locus, which encodes two critical CDK inhibitors $p 16^{I N K 4 a}$ and $p 15^{I N K 4 b}$ in up to $80 \%$ of malignant mesotheliomas..$^{29,30,33}$ This observation is also supported by recent gene expression studies. ${ }^{27,28}$ The deletion can be detected by FISH, which prompted some investigators to use this technique to evaluate body cavity fluids. ${ }^{34}$ Recognizing the difficulty of separating malignant from reactive mesothelial proliferations in some surgical pathology and effusion cytology samples, we sought to test the utility of this technique for aiding in resolution of this differential diagnosis. After more than 15 years of investigation, there are no established reliable immunohistochemical or molecular markers that can differentiate between benign mesothelial proliferations and diffuse malignant mesothelioma. A significance of recently recognized marker of malignancy, GLUT-1, in malignant mesothelial proliferations remains to be validated. ${ }^{24}$ Illei et $a l^{34}$ demonstrated that homozygous CDKN2A deletion detected by FISH is a very powerful technique for confirming the diagnosis 
of mesothelioma over reactive mesothelial cells in 12 of 13 patients with positive or suspicious cytology. In the same study, authors were able to demonstrate the lack of deletion in cytologically negative effusion specimens with atypical mesothelial cells confirming the low false-negative rate. Our study confirmed this observation on randomly selected pleural biopsies with malignant and benign reactive mesothelial proliferations. Similar to effusion cytology, the distinction between these two diagnostic possibilities could be extremely difficult, particularly when biopsy specimens are small and superficial. In our study, all pleural biopsies displaying reactive mesothelial proliferations in backgrounds of acute fibrinous pleuritis or chronic pleuritis were negative for 9p21 deletion, while the deletion was easily detected in a substantial number of mesotheliomas.

The proportion of malignant mesothelioma demonstrating the 9p21 deletion in our study $(67 \%$ for pleural and $25 \%$ for peritoneal) is similar to a PCR-based study reported by Hirao et $a l^{35}$ who found homozygous deletion in $22 \%$ of 45 analyzed mesotheliomas. It is known that other alterations may occur at this locus that cannot be detected by FISH. In the same study, 9\% of malignant mesotheliomas were found to have promoter hypermethylation and $2 \%$ were found to harbor a point mutation of the $p 16$ gene. ${ }^{35}$ We did not explore these two genetic alterations in our study because a different technical approach would be needed, but these alterations may have existed in cases included in our study. We chose to use FISH analysis in our study for several reasons. The FISH assay is available in many clinical laboratories, can be reliable performed on archival paraffin-embedded tissue and is relatively less expensive than other molecular assays. Another advantage of this technique over PCR-based assays is the ability to identify homozygous and hemizygous deletions. Furthermore, different tumor areas can be simultaneously analyzed and visualized.

We also noted differences in the frequency of homozygous 9p21 deletion between pleural and peritoneal mesotheliomas. This observation suggests that the frequencies of 9p21 deletions may be influenced not only by different genetic mechanisms and methods of detection, but also by some environmental factors. It is known that chromosome 9p represents a chromosomal region that is more prone to DNA damage by asbestos. ${ }^{36}$ It is interesting that in our study only 5 of 20 cases of peritoneal mesotheliomas displayed the 9p21 deletion and 2 of these cases had documented asbestos exposure. In the other cases, the exposure history was either negative or unknown. The level of exposure may be another explanation for lower frequency of detected deletions in this group. However, it is difficult to accurately evaluate relative asbestos exposure, either by questionnaire or fiber counting. It would be interesting to know whether deletion of 9p21 correlates with the type of asbestos fibers to which patients were exposed. It is known that crocidolite is the most pathogenic amphibole fiber, and it is possible that patients with peritoneal mesotheliomas were exposed to other types of fibers as well.

Our immunohistochemistry data confirmed a frequent loss of p16 immunoreactivity in pleural and peritoneal malignant mesotheliomas. In our study, homozygous deletion did not correlate with p16 protein expression in any of the studied groups, indicating that homozygous deletion is a mechanism for loss of protein expression in a subset of malignant mesotheliomas, or potentially a reflection of imperfect antibody specificity. Other mechanisms such as gene mutation, methylation or transcriptional and post-transcriptional factors may have a role in explaining the discrepant results. Alternatively, discrepancies between FISH and immunostaining results, particularly in cases showing homozygous deletion of 9p21 and diffuse p16 immunoexpression, could be a result of a false-positive FISH results caused by suboptimal hybridization.

In summary, our study suggests that FISH assessment of homozygous 9p21 deletion may be useful for ascertaining the nature of mesothelial proliferations. A discrepancy between p16 protein expression and homozygous deletion data suggests that other molecular mechanisms play a role in $p 16$ protein expression in mesothelial proliferations. Additional studies on larger series of patients are needed to evaluate the validity of this diagnostic approach.

\section{Acknowledgement}

We thank Kathleen Cieply, Tracy Mercuri, Carol Sherer, Kathleen Cumbie in the FISH laboratory and Kimberly Fuhrer, Developmental Laboratory of the Department of Pathology University of Pittsburgh Medical Center, for their technical assistance.

\section{References}

1 US-Canadian Mesothelioma Reference Panel:Churg A, Colby TV, Cagle P, et al. The separation of benign and malignant mesothelial proliferations. Am J Surg Pathol 2000;24:1183-1200.

2 Churg A, Roggli V, Galateau-Salle F, et al. Tumours of the pleura: mesothelial tumours. In: Travis WD, Brambilla E, Harris CC, Muller-Hermelink HK (eds). World Health Organization Classification of Tumours, Pathology and Genetics: Tumours of the Lung, Pleura, Thymus and Heart. IARC: Lyon, 2004, pp 128-136.

3 Galateau-Salle F, Brambilla E, Cagle PT, et al. Pathology of Malignant Mesothelioma. International Mesothelioma Panel. Springer-Verlag: London, 2006.

4 Churg A, Cagle PT, Roggli VL. Tumors of the Serosal Membranes. Atlas of Tumor Pathology Fascicle. ARP Press: Silver Spring, MD, 2006. 
5 IMIG Pathology Panel:Husain AN, Borczuk AC, Cagle PT, et al. Malignant mesothelioma: guidelines for pathologic diagnosis. Lung Cancer (in press).

6 Cagle PT. Pleural Histology. In: Light RW, Lee YCG (eds). Pleural Disease: An International Textbook. Arnold Publishers: London, 2003, pp 249-255.

7 Allen TC. Recognition of histopathologic patterns of diffuse malignant mesothelioma in differential diagnosis of pleural biopsies. Arch Pathol Lab Med 2005; 129:1415-1420.

8 Laqa AC, Allen T, Bedrossian C, et al. Diffuse malignant mesothelioma. In: Cagle PT (editor-in-chief) The Color Atlas and Text of Pulmonary Pathology. Lippincott Williams and Wilkins: New York, 2005, pp 143-148.

9 Khoor A, Whitsett JA, Stahlman MT, et al. Utility of surfactant protein B precursor and thyroid transcription factor 1 in differentiating adenocarcinoma of the lung from malignant mesothelioma. Hum Pathol 1999;30:695-700.

10 Granville LA, Younes M, Churg A, et al. Comparison of monoclonal vs polyclonal calretinin antibodies for immunohistochemical diagnosis of malignant mesothelioma. Appl Immunohistochem Mol Morphol 2005; 13:75-79.

11 Butnor KJ, Nicholson AG, Allred DC, et al. Expression of renal cell carcinoma-associated markers erythropoietin, CD10, and renal cell carcinoma marker in diffuse malignant mesothelioma and metastatic renal cell carcinoma. Arch Pathol Lab Med 2006;130: 823-827.

12 Galateau-Sallé F, Cagle PT. Non-malignant vs Malignant Proliferations on Pleural Biopsy. In: Cagle PT (ed). Diagnostic Pulmonary Pathology. Marcel Dekker: New York, NY, 2000, pp 555-567.

13 Cagle PT, Churg A. Differential diagnosis of benign and malignant mesothelial proliferations on pleural biopsies. Arch Pathol Lab Med 2005;129:1421-1427.

14 Kerrigan SA, Cagle PT, Churg A. Malignant mesothelioma of the peritoneum presenting as an inflammatory lesion: a report of four cases. Am J Surg Pathol 2003; 27:248-253.

15 Whitaker D, Henderson DW, Shilkin KB. The concept of mesothelioma in situ: implications for diagnosis and histogenesis. Semin Diagn Pathol 1992;9:151-161.

16 Henderson DW, Shilkin KB, Whitaker D. Reactive mesothelial hyperplasia vs mesothelioma, including mesothelioma in situ: a brief review. Am J Clin Pathol 1998;110:397-404.

17 Cury PM, Butcher DN, Corrin B, et al. The use of histological and immunohistochemical markers to distinguish pleural malignant mesothelioma and in situ mesothelioma from reactive mesothelial hyperplasia and reactive pleural fibrosis. J Pathol 1999;189: 251-257.

18 Mayall FG, Goddard H, Gibbs AR. p53 immunostaining in the distinction between benign and malignant mesothelial proliferations using formalin-fixed paraffin sections. J Pathol 1992;168:377-381.

19 Ramael M, Lemmens G, Eerdekens C, et al. Immunoreactivity for $p 53$ protein in malignant mesothelioma and non-neoplastic mesothelium. J Pathol 1992;168: 371-375.

20 Cagle PT, Brown RW, Lebovitz RM. p53 immunostaining in the differentiation of reactive processes from malignancy in pleural biopsy specimens. Hum Pathol 1994;25:443-448.
21 Wolanski KD, Whitaker D, Shilkin KB, et al. The use of epithelial membrane antigen and silver-stained nucleolar organizer regions testing in the differential diagnosis of mesothelioma from benign reactive mesothelioses. Cancer 1998;82:583-590.

22 Attanoos RL, Griffin A, Gibbs AR. The use of immunohistochemistry in distinguishing reactive from neoplastic mesothelium. A novel use for desmin and comparative evaluation with epithelial membrane antigen, p53, platelet-derived growth factor-receptor, P-glycoprotein and Bcl-2. Histopathology 2003;43:231-238.

23 Saad RS, Cho P, Liu YL, et al. The value of epithelial membrane antigen expression in separating benign mesothelial proliferation from malignant mesothelioma: a comparative study. Diagn Cytopathol 2005; 32:156-159.

24 Kato Y, Tsuta K, Seki K, et al. Immunohistochemical detection of GLUT-1 can discriminate between reactive mesothelium and malignant mesothelioma. Mod Pathol 2007;20:215-220.

25 Musti M, Kettunen E, Dragonieri S, et al. Cytogenetic and molecular genetic changes in malignant mesothelioma. Cancer Genet Cytogenet 2006;170:9-15.

26 Illei PB, Rusch VW, Zakowski MF, et al. Homozygous deletion of $C D K N 2 A$ and codeletion of the methylthioadenosine phosphorylase gene in the majority of pleural mesotheliomas. Clin Cancer Res 2003;9: 2108-2113.

27 Singhal S, Wiewrodt R, Malden LD, et al. Gene expression profiling of malignant mesothelioma. Clin Cancer Res 2003;9:3080-3097.

28 Lopez-Rios F, Chuai S, Flores R, et al. Global gene expression profiling of pleural mesotheliomas: overexpression of aurora kinases and $p 16 / C D K N 2 A$ deletion as prognostic factors and critical evaluation of microarray-based prognostic prediction. Cancer Res 2006;66:2970-2979.

29 Xio S, Li D, Vijg J, et al. Co-deletion of p15 and p16 in primary malignant mesothelioma. Oncogene 1995;11: 511-515.

30 Prins JB, Williamson KA, Kamp MM, et al. The gene for the cyclin-dependent-kinase-4 inhibitor, CDKN2A, is preferentially deleted in malignant mesothelioma. Int J Cancer 1998;75:649-653.

31 Walts AE, Lechago J, Bose S. P16 and Ki67 immunostaining is a useful adjunct in the assessment of biopsies for HPV-associated anal intraepithelial neoplasia. Am J Surg Pathol 2006;30:795-801.

32 Papachristou DJ, Goodman MA, Cieply $\mathrm{K}$, et al. Comparison of allelic losses in chondroblastoma and primary chondrosarcoma of bone and correlation with fluorescence in situ hybridization analysis. Hum Pathol 2006;37:890-898.

33 Cheng JQ, Jhanwar SC, Klein WM, et al. p16 alterations and deletion mapping of 9p21-p22 in malignant mesothelioma. Cancer Res 1994;54:5547-5551.

34 Illei PB, Ladanyi M, Rusch VW, et al. The use of $C D K N 2 A$ deletion as a diagnostic marker for malignant mesothelioma in body cavity effusions. Cancer 2003; 99:51-56.

35 Hirao T, Bueno R, Chen C-J, et al. Alterations of the p16(INK4) locus in human malignant mesothelial tumors. Carcinogenesis 2002;23:1127-1130.

36 Borczuk AC, Taub RN, Hesdorffer M, et al. P16 loss and mitotic activity predict poor survival in patients with peritoneal malignant mesothelioma. Clin Cancer Res 2005;11:3303-3308. 